\title{
Towards Intelligent Chatbots for Customer Care - Practice-Based Requirements for a Research Agenda
}

\author{
Carl Corea \\ Institute for IS Research \\ University of Koblenz-Landau \\ ccorea@uni-koblenz.de
}

\author{
Patrick Delfmann \\ Institute for IS Research \\ University of Koblenz-Landau \\ delfmann@uni-koblenz.de
}

\author{
Sabine Nagel \\ Institute for IS Research \\ University of Koblenz-Landau \\ snagel@uni-koblenz.de
}

\begin{abstract}
Chatbots bear a great potential to save efforts and costs in customer care through service automation. Current results are however still at an early stage in functionality and not widely attainable. Here, developing a new form of intelligent chatbots is a current challenge still under review. While there have been numerous proposals for future work, virtually all agenda-setting contributions are solely based on scientific literature. This is unsatisfactory from both an academic and practical perspective, as the industrial view on the future of chatbots seems to be neglected. Therefore, this work explores how professional experts see the future of intelligent chatbots for customer care and suggests how practice can guide research based on an expert panel with 17 industrial partners. Our work identifies research opportunities based on the demands and views of key practitioners by pin-pointing expected trends. Furthermore, based on the expert opinions, we derive guidelines for organizations which state key factors that should be considered in the development or adoption of chatbots in customer care.
\end{abstract}

\section{Introduction}

Customer care is a central topic for todays organizations and comprises methods and techniques for offering customer service or managing long-term customer relations and customer retention [1, 2, 3]. In light of the digital transformation, companies are currently investing enormous resources into the digital innovation of customer care in order to stay competitive [4]. An important factor here is the increasing number of online customer service requests, e.g. over social-media or company websites [3]. For example, a recent study by $\mathrm{Hu}$ et al. (2019) reports that $67 \%$ of U.S. online retail customers have ushered a service request via social-media. Also, next to the large number of requests, changed customer demands such as quick response rates impose current challenges [5]. For example, a recent study by Baer (2016) reports that the average response time to customer service requests via social media was $6.5 \mathrm{~h}$, whereas $75 \%$ of customers expected a response time of less than an hour.

To conquer such problems, chatbots have recently received major attention from practice. Chatbots are text-based and autonomous dialogue systems that can support the customer in predefined scenarios [6]. For example, a chatbot could autonomously help customers with a request, such as providing status information for an order. Intuitively, chatbots consequently bear a great potential for automating customer care. Next to an increased efficiency and a reduction of manual effort, recent studies also suggest that faster response rates (i.e., instantaneously via chatbots) have a significant positive impact on the customer satisfaction [3, 7]. In turn, chatbots can therefore contribute towards customer delight by positively exceeding customer expectations [7]. The study by Zarouali et al. (2018) could show that customer delight via chatbots can also contribute towards brand loyalty and customer retention.

Despite these potential benefits in efficiency and customer satisfaction, the current state-of-the-art in chatbot technologies is still at an early stage, yielding impediments for the practical application of chatbots in customer care. For example, as current chatbot dialogues strongly rely on predefined phrases and results from natural language parsing (NLP), chatbots often reach their boundaries, yielding unsatisfactory service or trapping customers in conversation loops.

Here, intelligent chatbots are needed, that can predict if they will not be able to handle the customer case correctly and the case should be transferred to a human employee. Intelligent chatbots should also be able to integrate and handle data from different systems, to create a unified viewpoint for customer care and a more advanced, seamless customer experience. These current challenges call for future research in this emerging field, in order to increase the value of chatbots for companies. To this aim, there have been some recent research agendas, identifying needed research 
topicalities (cf. Section 2). However, existing research agendas are based on reviews of scientific literature and/or a scientific viewpoint. In turn, demands and insights from practice are not represented in current efforts towards research agendas. On the contrary, the aim of this work is therefore to identify needed research for intelligent chatbots, based on the demands and views of key practitioners. This work is the result of a requirements analysis phase in a research project on intelligent chatbots we are currently conducting with industrial partners. Here, we conducted interviews with experts working in customer service, customer relationship management, and marketing to gain insights on a practitioners' view on the future of intelligent chatbots in customer care.

The contribution of this work is as follows. First, we motivate the need for a practice-based research agenda by means of a literature review (Section 2). Second, we present the results of our interview analysis, which reveals important trends that are expected from professionals in customer care and should therefore be attended to (Section 3-4). Then, based on the interview analysis and identified trends, we propose a novel practice-based research agenda, as well as practical guidelines for implementing and adopting chatbots in customer care (Section 5).

\section{Research Background}

This Section introduces current limitations for chatbots in customer care and provides an overview of current research agendas in this field.

\subsection{Chatbots}

Chatbots are based on predefined structures, i.e., a directed graph of so-called dialogue points which constitute responses to questions, as shown in Figure 1 $[8,9]$. Given a customer input, the chatbot is supposed to

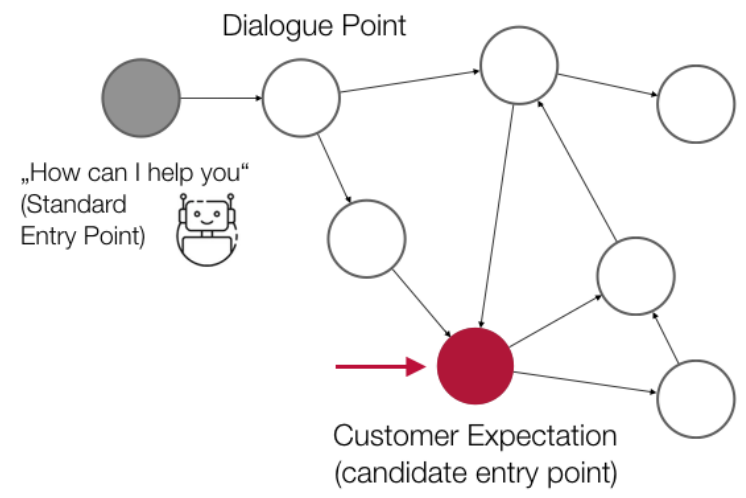

Figure 1. Exemplary dialogue structure. correctly navigate through the individual dialogue points in order to subsequently handle the request. Currently, this processing of customer input is conducted by means of natural language processing (NLP) [8]. Yet, current NLP techniques have not reached a sufficient level of maturity to route the chatbot dialogue in a satisfactory manner $[10,11]$. Also, the customer has to enter his request in the first place and possibly has to navigate through a cumbersome dialogue. Instead, it would be better if chatbots could recognize the customer behavior, understand exactly which type of help the customer needs, and determine a fitting entry point in the chatbot dialogue, i.e., the dialogue could start directly fitting the customer request as hinted in Figure 1. Here, intelligent chatbots are needed, that can analyze and predict customer behavior in order to be able to determine these entry points in the dialogue structure.

Furthermore, existing chatbot solutions are currently not process-aware [6]. That is, while they can navigate through their predefined dialogue structure, they cannot interrelate the individual points of the structure. In result, the chatbot has no understanding of how the current dialogue point is connected to company activities or other systems. This can result in frustrating problems for the customer, e.g., loops in the dialogue, and wastes great potential to integrate other systems or services with the chatbot dialogue. These current limitations call for further development of intelligent chatbots.

\subsection{Agenda Setting}

As a basis for this work, we wanted to gain an oversight of current research agendas in the field of chatbots and customer care. We therefore conducted a literature search following the process proposed by vom Brocke et al. (2009). We conducted a keyword-based search as suggested in [12, 13, 14], using the keywords "research agenda" and "chatbots". We queried 6 pertinent literature databases to warrant a broad view on existing works, namely ACM digital library ${ }^{1}$, Springer Link $^{2}$, Emerald Insight ${ }^{3}$, AISel electronic library ${ }^{4}$, Science Direct ${ }^{5}$ and IEEE Xplore ${ }^{6}$. The query was defined s.t. the work (any field) had to contain the above keyword combinations. As a result, our keyword based search yielded 58 results. We considered a paper to be relevant if it presented a form of research agenda for the topics of chatbots. Here, we could identify 11 relevant

\footnotetext{
${ }^{1}$ https: //dl.acm.org/

2https://link.springer.com/

${ }^{3}$ https: // www. emeraldinsight.com/

${ }^{4}$ https://aisel.aisnet.org/

5 https://www. sciencedirect.com/

${ }^{6}$ https://ieeexplore.ieee.org/
} 
works. Our literature search contains results up to April of 2019.

Table 1 shows the results of our literature search. While there exist various research agendas for chatbots from a technical perspective, we see that there are no research agendas investigating the link between chatbots and customer care (Research gap 1). Therefore, this work contributes to the field by distilling a research agenda with a joint scope of these topics. Furthermore, our literature search reveals that existing agendas strongly ignore a practice-based view (Research gap 2 ). While of course we do not question the value of these works, we argue that agenda-setting exploratory research should involve both academia and industry, in order to guide research from the perspective of practical-relevance. The aim of this work is therefore not to replace existing agendas, but rather to advance the field by providing a practice-based view. Here, our work identifies research opportunities based on the actual needs and experiences of experts in the field of customer care, marketing, and chatbots.

\begin{tabular}{l|l|l|l}
\hline Source & {$[$ Ref] } & Topic & Basis \\
\hline Riccardi (2014) & {$[15]$} & $\begin{array}{l}\text { Design of } \\
\text { Chatbot Systems }\end{array}$ & Literature Review \\
\hline Io et al. (2017) & {$[16]$} & $\begin{array}{l}\text { Design of } \\
\text { Chatbot Systems }\end{array}$ & Literature Review \\
\hline Bolton et al. (2018) & {$[17]$} & $\begin{array}{l}\text { Customer } \\
\text { Experience }\end{array}$ & $\begin{array}{l}\text { Literature Review, } \\
\text { Case Studies }\end{array}$ \\
\hline Folstad et al. (2018) & {$[18]$} & $\begin{array}{l}\text { Design of } \\
\text { Chatbot Systems }\end{array}$ & Literature Review \\
\hline Rzepka et al. (2018) & {$[19]$} & $\begin{array}{l}\text { User Interaction } \\
\text { with Chatbots }\end{array}$ & Literature Review \\
\hline Siddike et al. (2018) & {$[20]$} & $\begin{array}{l}\text { Trust in } \\
\text { Chatbot Systems }\end{array}$ & Literature Review \\
\hline Tavanapour et al. (2018) & {$[21]$} & $\begin{array}{l}\text { Design of } \\
\text { Chatbot Systems }\end{array}$ & Literature Review \\
\hline Wirtz et al. (2018) & {$[22]$} & $\begin{array}{l}\text { Design of } \\
\text { Chatbot Systems }\end{array}$ & Literature Review \\
\hline Diederich et al. (2019) & {$[9]$} & $\begin{array}{l}\text { Design of } \\
\text { Chatbot Systems }\end{array}$ & Literature Review \\
\hline Lechler et al. (2019) & {$[23]$} & $\begin{array}{l}\text { Design of } \\
\text { Chatbot Systems }\end{array}$ & $\begin{array}{l}\text { Literature Review, } \\
\text { Tool Analysis }\end{array}$ \\
\hline Piccolo et al. (2019) & {$[24]$} & $\begin{array}{l}\text { Design of } \\
\text { Chatbot Systems }\end{array}$ & Literature Review \\
\hline
\end{tabular}

Table 1. Overview of existing research agendas for chatbots.

\section{Methodology}

This study is part of a research project on intelligent chatbots. The project brings together multiple marketing/consulting agencies specialized in customer care, a collection of respesctive industrial clients, and an academic viewpoint with a strong background in predictive analytics. The 14 participating companies range from small-/medium enterprises to large enterprises (up to 7500 employees) and represent diverse fields with a high amount of customer interaction and demand for customer service, such as the energy industry, e-commerce, sport- and event management or the insurance industry.

We currently see a lack of practice-based requirements in current research agendas on chatbots based on our literature review.

Consequently, the research aim of this work is to identify needed research for intelligent chatbots, based on the demands and views of key practitioners.

To this aim, we follow an expert panel approach as proposed in $[25,26]$. We see this methodology as highly appropriate for our research aim, as it allows to collect "rich and indepth information about the experiences of individuals" as a basis for identifying key factors that are perceived to be important by key practitioners for future research [26][p.319]. Figure 2 shows the individual phases of our research.

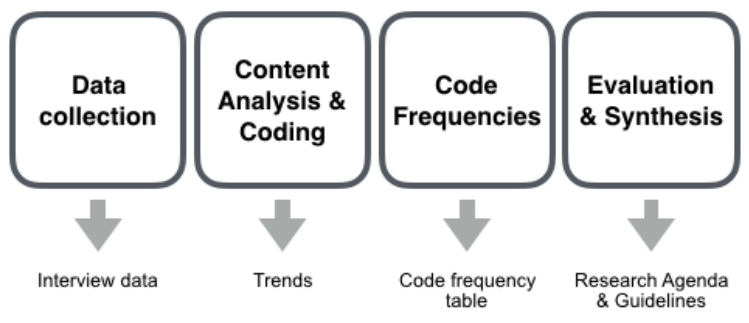

Figure 2. Overview of research methodology.

For data collection, we conducted semi-structured interviews with 17 experts in the field from January-February of 2019. The experts' profile was as follows (we denote the individual experts with a running id for future reference): We interviewed consultants from a total of six different marketing/consulting agencies (E1, E2, E3, E4, E10, E12, E13) and practitioners from a large online comparison portal (E4), an online streaming platform (E11), two vendors in the energy industry (E6, E7, E17), a large insurance company (E8), an international car rental company (E9), a German premier-league soccer club (E15), an event management company (E16), and a large newspaper publisher (E14). All experts work in fields with a strong demand for customer care and represent a wide range of technical or managerial backgrounds. The findings of this work are based on the central question of our semi-structured interviews, namely "How do you expect chatbots in customer care to evolve in the (near) future, and what needs to change?".

The interviews were conducted individually to avoid peer pressure and distortion. To ensure internal validity, each interview was conducted by (the same) three researchers present, always following the 
same interview protocol. To warrant measurement validity, the answers were regularly repeated during the interview and clarified by specific inquiries in case of uncertainties. Also, each researcher created an individual transcript to implement investigator triangulation [27]. To promote reliability, the transcripts were then compared and discussed to create an aggregated data source.

This interview data was then analyzed following the coding process proposed in [28]. Here, topic and structural coding was used to represent the individual answers as concepts and subsequently to iteratively aggregate common themes. In the scope of quality assurance, the coding was performed independently. Identified codes were then discussed, conceptually matched and refined. Also, a coding frequency table was created to assess the perceived degree of importance for individual themes.

Finally, we identified trends that were perceived as important by the experts based on an evaluation and synthesis of our data analysis.

\section{Results}

Based on our interview analysis, we identify six trends which the experts expect in the future evolution of chatbots for customer care, shown in Table 2. As an overview, the trends are directed towards two general phases, namely the interaction between the chatbot and the customer (dialogue phase), and a phase before the dialogue between customer and chatbot has started, the so-called (pre-dialogue phase). The pre-dialogue phase is geared towards analyzing user behavior in order to predict that a customer requires help. In case of such a prediction, the goal is also to understand the customer intention and thus proactively determining a fitting entry point in the chatbot dialogue structure. In this way, intelligent chatbots can offer individual customer support (i.e., the customer does not have to navigate through a cumbersome dialogue, but rather the chatbot can determine a fitting entry point in the dialogue structure). Interestingly, the experts suggest that the pre-dialogue phase bares even greater potential, as it is currently the most neglected aspect according to the panel. During the actual dialogue phase, experts stated trends related to better, more intelligent chatbots that can handle requests more adequately and bridge the customer service request with other company systems or employees.

In the following, we detail the six trends that are expected for chatbots in customer care by the expert panel.

\begin{tabular}{l|c}
\hline Trend & Expert Count \\
\hline Chatbots as central orchestration tools & 17 \\
\hline $\begin{array}{l}\text { Process-Aware chatbots and } \\
\text { Customer Process Management }\end{array}$ & 16 \\
\hline Predictive X & 15 \\
\hline $\begin{array}{l}\text { Stronger tie between employee } \\
\text { and chatbot }\end{array}$ & 14 \\
\hline $\begin{array}{l}\text { Need for lower barriers in } \\
\text { chatbot adoption }\end{array}$ & 12 \\
\hline $\begin{array}{l}\text { More awareness of } \\
\text { data privacy }\end{array}$ & 6 \\
\hline
\end{tabular}

Table 2. Overview of the expected trends in chatbots for customer care, according to the expert panel $(\mathrm{N}=17)$.

\subsection{Chatbots as Central Orchestration Tools}

Most prominently, the experts indicated that many companies today still view chatbots as simple dialogue systems with little or no access to or knowledge of other company systems [E1, E2, E4, E10]. Here, the experts strongly suggest that a paradigm shift is crucial, shifting the perception of chatbots from dialogue systems to the de facto orchestration tool for companies [E1, E2, E5, E6, E12, E13]. The experts unanimously expect chatbots to become a new layer directly between the customer and company systems and activities, serving as a central platform to orchestrate company systems and/or trigger further company processes. Here, "the chatbot should become an assistant to the customer, which is aware of how the customer request should be forwarded and handled by the socio-technical company environment" [E3, Head of Client Services, Marketing/Consulting]. Experts also unanimously agreed that data integration is the biggest challenge for chatbots that needs to be addressed in the near future. E9 stated that "customer care processes typically include vast amounts of island solutions" [E9, head of social media, car-rental services]. E1 added that they even see situations where different departments in one company are using different tools for the same tasks [E1, CEO, Marketing/Consulting]. Multiple experts also named the problems of island solutions, but hinted that this might be a structural/organizational problem resulting from company growth or mergers and will not be fixable any time soon, thus demanding a stronger focus on creating unified views to integrate data [E2, E6, E7, E8, E9, E13]. With companies using heterogeneous systems, e.g. different tools for process management or customer relationship management, companies need to focus on creating a unified view to allow chatbots to understand and interact with this data [E1, E5, E10, 
E12, E13]. This could allow chatbots to understand the context of the customer request, which was perceived as an important means for the development of intelligent chatbots. In return, chatbots should not be limited by a predefined dialogue structure, but should be evolved into systems for orchestrating customized customer service, e.g. by accessing previous orders by a customer and enriching the dialogue with fitting, proactive service. For example, if a customer has recently made an order, and it appears the customer is browsing the website trying to find information on this order, the chatbot should infer that there might be something wrong with this specific order and engage the customer proactively by asking whether everything is alright with the respective order, as well as a list of fitting options such as "do you want me to track your order for you?". The COO of a marketing agency (E2) also added that companies are currently also having huge difficulties in synchronizing and maintaining omni channel chatbot systems, i.e., chatbot communication across different platforms or devices. With customers demanding customer care ubiquitously, maintaining a seamless user experience becomes difficult to implement. Here, E2 amongst others sees a strong need for creating central chatbot solutions that serve as a unified orchestration tool [E1, E2, E5, E9, E13].

\subsection{Process-Aware Chatbots and Focus on Customer Process Management}

Currently, chatbots follow predefined dialogue structures but have no awareness of what the process actually means, and how individual dialogue points are connected to other company processes [6]. Experts unanimously perceived process-awareness as a critical success factor for future chatbots as orchestration tools. Also, experts indicated that they expect the focus of companies to expand from Business Process Management (BPM) to Customer Process Management (CPM) [E1, E2, E3, E4, E8, E9, E10, E14, E17]. In BPM, "customers are often modeled as a black box" [E3, Head of Client Services, Marketing/Consulting], which serves merely as an input to trigger the well-defined company activities. Here, experts see the need to actually analyze, understand and model the customer processes [E1, E3, E4, E6, E9, E12]. "Business processes should be modeled around the customer process, not the other way around" [E6, Head of Innovation- and Change management, energy industry]. This could help to understand the activities of customers and apply amenities of process management to improve the customer experience and the perceived efficiency for the customer. Experts saw it as essential to assist customers in their activities and lower the manual effort customers have to invest [E1,E6,E9]. For example, when a customer visits a company website to file an application for a loan, systems should automatedly create customized online forms, pre-filled with relevant information which was gathered from company databases to reduce the manual effort for the customer.

\subsection{Predictive $X$}

Experts indicated a strong interest in adopting machine learning techniques to advance chatbot technology. The role of different predictive capabilities such as risk prediction, activity prediction or case-outcome prediction (in the following denoted as predictive $X$ ), was seen as a core driver for intelligent chatbots and customer care. Here, predictive $\mathrm{X}$ is expected to revolutionize both the dialogue phase, as well as the pre-dialogue phase, which is widely perceived as underdeveloped by the experts.

In the pre-dialogue phase, (customer) process-aware chatbots could predict the intention of the user and thus offer a proactive and individual support. Through a prediction based on customer behavior, the chatbot could directly show customized and relevant user prompts, instead of engaging all customers in the same starting point. For example, customers would not have to start with a generic chatbot dialogue (e.g., "how can I help you") and navigate through a bothersome dialogue, but could proactively be picked up with a support matching their needs (e.g. "can I help you to print a return label for your order XY?"). Again, data integration and context-awareness were seen as important future topics to allow for predictive $\mathrm{X}$ [E2, $\mathrm{E} 5, \mathrm{E} 8, \mathrm{E} 9, \mathrm{E} 12, \mathrm{E} 13]$. The panel expects that this form of proactively engaging customers will be implemented by organizations as a way to reduce manual effort for the customer, and a way to potentially increase customer satisfaction and create customer delight by means of proactive/surprising service. A lead developer (E5) added that investing in predictive analysis in the pre-dialogue phase in order to understand customer needs (instead of starting with a generic entry point for all customers) is crucial to allow chatbots to handle customer care requests correctly, as "the current way of determining which dialogue point to show based on the user input [(String)] and NLP-techniques is insufficient, and [he does] not see this changing any time soon" [E5, Lead developer, online-comparison portal]. Experts also emphasized the opportunity to segment customers by means of predictive $\mathrm{X}$ in the pre-dialogue phase. That is, intelligent chatbots should be able to analyze 
the customers and their behavior in order to predict user intentions and prioritize the customer care cases, e.g., identify high-priority cases to implement customer segmentation and provide an informed basis to support organizations in managing their human resources.

Also, predictive $\mathrm{X}$ was seen as an important topic during the actual chatbot dialogue (dialogue phase). Process-aware chatbots were seen as a central driver in this context, as process-awareness allows chatbots to monitor their own performance with the customer through predictive monitoring. This allows chatbots to recognize, that they will not be able to handle the current customer case correctly, thus a hand-off to a human employee could be initiated on-demand. While experts agree that predictive $X$ will play an important role in predictive monitoring during the dialogue phase, the biggest change is expected in using predictive $\mathrm{X}$ to analyze and understand customer behavior in the pre-dialogue phase, which is perceived as "practically non-existent in current tools" [E2,COO, Marketing/Consulting].

\subsection{Stronger Tie between Employees and Chatbots}

Experts see the hand-off of customer support requests from the bot to the human employee as a highly important topic. Again, predictive $X$ is seen as a key trend to facilitate such a hand-off [E1, E2, E3, E5, E6, E9, E12, E13]. A shared consensus here is that chatbots are not expected to replace human employees. Instead, the role of chatbots is envisioned to be an assistant to human employees, which can reduce effort by automating sub-tasks, pre-prioritizing customer care cases or provide capabilities for companies to increase the efficiency and effectiveness of using case data to understand customer cases correctly [E1, E3, E7, E8, E9, E14, E17]. First, chatbots should be able to initiate an on-demand hand-off to human employees, based on alarm-based predictive monitoring during the dialogue phase, i.e., the chatbot should be able to recognize when it will not be able to handle the case correctly. Here, E1,E2 and E5 expressed the importance of developing new means for knowledge transfer between chatbots and employees during this hand-off. Companies need to find new ways to illustrate what the chatbot has already discussed with the customer and means to visualize the resp. case data such that the human employee can seamlessly continue the customer case in an efficient manner, and does not bother customers with repeated questions. "The chatbot may not have been able to help the customer, but it still had a conversation with the customer. We currently see that this information is however often lost during these changes of format, yet the customer demands for a seamless experience." [E2, $\mathrm{COO}$, Marketing/Consulting]. Prescriptive methods are also seen as an important challenge for companies in this context. For example, to promote efficient knowledge transfer, chatbots should "present assessments and action proposals based on the analysis of their preceding dialogue with the customer [to guide employees]" [E5, Lead Developer, Online Comparison Platform].

\subsection{Need for Lower Barriers in Chatbot Adoption}

The experts perceive a strong need for lower barriers in chatbot adoption, as they feel that companies are currently challenged to understand their data and implement machine learning technologies. E10 commented that "our clients, especially small and medium enterprises, simply do not have the resources to hold this knowledge available." [E10, Principal Consultant, Marketing/Consulting]. This is affirmed by the opinion of E1, who remarked that "machine learning is a hot-, yet still difficult topic for many of our customers." [E1, CEO, Marketing/Consulting]. In the context of chatbots for customer care, experts expect a rise of tools to guide companies in the implementation and maintenance of chatbot systems in a step-by-step manner without extensive knowledge of machine learning or programming skills, as well as dashboards to monitor chatbot systems. E1 also commented that "we saw huge success in tools that allowed our customers to create content like giveaways without programming skills for social media. The same can be expected for chatbots.”. Experts E2 and E5 especially emphasized the need for intuitive user interfaces that allow to create chatbot dialogues and to define APIs to other systems and plugins in a step-by-step manner. The need for metrics and dashboards to facilitate advanced monitoring was broadly viewed as an important trend in this context.

\subsection{More Awareness of Data Privacy}

Finally, experts also uttered concerns about the future of chatbots in customer care regarding data privacy. While intelligent chatbots could use predictive $\mathrm{X}$ and data integration to improve services, these functionalities are broadly based on tracking customer behavior and/or processing customer data. Especially E8,E9 and E13 reported that the awareness of customers for data privacy and tracking has recently changed, for example due to the rise of so-called cookie-banners or recent data-leak scandals. Here, companies need to find 
an adequate balance between involving the customer in customizing data privacy preferences while ensuring a sufficient basis for intelligent chatbot functionalities. Interestingly, E8 saw this partially as a problem beyond the scope of companies but rather dependent also on politics and regulations and remarked that "ensuring compliant processes and technologies will become one of the biggest challenges for us in the context of digital transformation" [E8, Organization Manager, Insurance Industry].

\section{Discussion}

In this section, we propose propose needed future research distilled from the practice-based requirements of our interview analysis, and guidelines for executives based on the suggestions of the expert panel.

\subsection{Practice-based Requirements for a Research Agenda}

From our interview analysis and the expected trends, we could identify much needed future work regarding two core aspects, namely technical aspects (e.g., machine learning approaches) and strategic aspects (e.g., new challenges arising from a new form of communication between employees and chatbots). Consequently, we distilled a corresponding, practice-based research agenda, presented in Table 3. In the following, we outline how this agenda could contribute towards the expected trends for intelligent chatbots.

From a technical viewpoint, we can identify research needed regarding foundational and engineering aspects, which includes the design and development of methods, techniques, and prototypes or technical artifacts for chatbots in customer care.

The overall gist of these technical-related aspects is the need for more data research, and a better integration of results from the field of data science into chatbot research. Topics in data research can be linked to the expected trends, e.g., data mining (Trend 1, Trend 3), machine learning (Trend 3, Trend 4) or data analytics (Trend 2, Trend 3). Our experts emphasized the need for data integration as a basis for evolving chatbots into central orchestration platforms. Here, data from heterogeneous systems has to be processes in order to create a unified viewpoint and offer more advanced features, such as managing omni channel communications or utilizing context to analyze and predict customer behavior.

\begin{tabular}{|c|c|}
\hline Trend & Research needed \\
\hline $\begin{array}{l}1 \text { - } \\
\text { (Orchestration) }\end{array}$ & $\begin{array}{l}\text { - Data integration of heterogeneous customer } \\
\text { relationship management systems } \\
\text { - How to utilize contextual data for } \\
\text { diagnostic/predictive analytics (Process case } \\
\text { interaction) } \\
\text { - Handling ubiquitous and omni-channel } \\
\text { communication with customers (Synchronization) } \\
\text { - How to integrate chatbot systems with existing } \\
\text { - enterprise architecture } \\
\text { - Best-practices and case-studies on how to evolve } \\
\text { chatbots from dialogue to orchestration systems } \\
\text { (Change Management) } \\
\text { - Design and implementation of chatbot standards } \\
\text { and APIs }\end{array}$ \\
\hline $\begin{array}{l}2 \text { - } \\
\text { (Process- } \\
\text { Awareness) }\end{array}$ & $\begin{array}{l}\text { - Methods, techniques and tools for process-aware } \\
\text { chatbots } \\
\text { - Reducing (manual) effort for customers } \\
\text { through assisting customers in activities } \\
\text { and decision-making (Customer Process } \\
\text { Management/Customer Process Automation) } \\
\text { - Guidelines for balancing exploitation and } \\
\text { exploration in customer processes (Ambidextrous } \\
\text { CPM) } \\
\text { - Creating customer delight using chatbots } \\
\text { - Joint creation of process value with customers } \\
\text { - Strategic alignment of BPM, CPM and chatbots } \\
\text { (value-driven CPM) }\end{array}$ \\
\hline $\begin{array}{l}3 \text { - } \\
\text { (Machine- } \\
\text { Learning) }\end{array}$ & $\begin{array}{l}\text { - ML methods and techniques to predict user } \\
\text { behavior } \\
\text { - New data sources to enrich predictions (context, } \\
\text { tone-aware chatbots) } \\
\text { - Proactive conversation initiation } \\
\text { - Outcome-oriented predictions for autonomous } \\
\text { monitoring }\end{array}$ \\
\hline $\begin{array}{l}4 \text { - } \\
\text { (Bots and } \\
\text { Employees) }\end{array}$ & $\begin{array}{l}\text { - On-demand handoff between chatbot and } \\
\text { employee, initiated by chatbots (alarm-based } \\
\text { predictive process monitoring) } \\
\text { - Knowledge transfer between chatbot and employee } \\
\text { (Metrics and Visualization) } \\
\text { - Prescriptive assistance of employees during } \\
\text { handoff } \\
\text { - Multi-employee customer support including } \\
\text { chatbots } \\
\text { - How to introduce and embrace a new culture of } \\
\text { "robotic" colleagues }\end{array}$ \\
\hline $\begin{array}{l}5- \\
\text { (Adoption) }\end{array}$ & $\begin{array}{l}\text { - Maturity models to promote the adoption of chatbot } \\
\text { technology } \\
\text { - Success Stories/Case Studies to share knowledge } \\
\text { and leverage chatbot adoption } \\
\text { - How to help companies to monitor customer care } \\
\text { using chatbots (Dashboards) } \\
\text { - How to create value from chatbot data (Effective } \\
\text { use of insights, Visualizations) } \\
\text { - Assisted implementation of chatbots }\end{array}$ \\
\hline $\begin{array}{l}6- \\
\text { (Privacy) }\end{array}$ & $\begin{array}{l}\text { - How to balance functionality vs. privacy } \\
\text { - Implementing governance and compliance } \\
\text { management } \\
\text { - How to include the customer in configuring privacy } \\
\text { preferences } \\
\text { - How to capture, maintain and implement relevant } \\
\text { regulatory policies (Knowledge Management, } \\
\text { Business Rules Management) }\end{array}$ \\
\hline
\end{tabular}

Table 3. Research Agenda: Overview of potential research avenues for intelligent chatbots. 
Moreover, experts expect a paradigm shift from business process management to customer process management (Trend 2, Trend 3). That is, while business process management will still be important to increase efficiency and ensuring compliant company activities, more research on how to understand and utilize customer processes to improve the customer experience is needed (e.g., in the scope of process mining and analysis).

Also, the importance of further evolving machine learning technologies in chatbot research is seen as highly important by experts (Trend 1, Trend 2, Trend 3, Trend 4). While there have been good advancements in the actual chatbot dialogue phase, solutions for process-aware chatbots or chatbots which can analyze user behavior to offer a proactive and customized support are strongly lacking, which imposes great research opportunities. Here, more research on predictive and prescriptive analytical techniques is needed.

In general, companies need more design-based research that focuses on the creation of methods, techniques and artifacts for chatbots in customer care. Especially regarding the hand-off between the chatbot and a human employee, experts indicated a strong need for new or improved artifacts, offering prescriptive recommendations in order to foster seamless user experience. Also, the barrier for chatbot adoption needs to be lowered according to our expert panel. New means which allow companies to implement and maintain chatbots without a deep technical knowledge need to be investigated. Again, helping companies to monitor and understand their data to create value through innovation offers much room for future research.

From a strategic viewpoint, we can identify research needed regarding a better understanding of managerial aspects in the implementation and adoption of intelligent chatbot technology.

Overall, potential research topics from this perspective comprise the areas of change management, strategic alignment, work culture, customer relationship management, and creating value for companies through chatbot systems.

As chatbots in customer care are still at an early stage, more research to advocate chatbot adoption is needed. For example, maturity models to guide companies in chatbot adoption or success stories to share knowledge could help to raise awareness and leverage chatbot adoption. In this context, helping companies to understand how new chatbot technology can be aligned and integrated with existing systems and enterprise architecture is strongly needed. Also, a new work culture of co-existent human and artificial employees opens research avenues for cultural and ethical investigations.

Through a focus on customer process management, future work should also be directed towards helping companies understand, model, and improve customer processes, e.g., by balancing explorative and exploitative approaches. Intelligent chatbots also open new opportunities for research on customer relationship management and customer satisfaction, e.g., the relation of chatbots and customer delight.

Finally, new forms of chatbot technology introduce new data analytics problems for companies. Future research is thus needed on how to create value from this data for companies. With a stronger focus on chatbots as central orchestration systems, monitoring (potentially automated) customer care also becomes increasingly important. Companies thus need to be supported in understanding the data created by chatbot systems as a basis for the innovative and sustainable development of systems and organizations.

To conclude, intelligent chatbots give rise to a diverse collection of research avenues. The proposed research agenda in Table 3 suggests how future research can contribute to the trends expected by the expert panel. As mentioned, it is not our goal to replace existing research agendas, but rather to identify research avenues seen as important by experts in the field, in order to further promote the integration of scientific results and practice.

\subsection{Guidelines for Executives}

Based on the experiences and expected trends by the expert panel, some practical guidelines for customer care with intelligent chatbots can also be identified.

Having the right mindset. Many experts from consulting companies reported that expectations of AI and chatbots are often biased by science-fiction. Companies should realize that chatbots/AI must not be seen as self-aware or self-learning systems, but rather that chatbots are systems that foster the automation of predefined activities. Organizations should have realistic expectations and must strive to identify and understand customer care processes as a basis for chatbot development. Here, respective budgets have to be allocated to allow for such an analysis and design phase, as this is the core basis for chatbot systems and thus customer care. If implemented correctly, chatbots can function beyond "simple" dialogue systems, by providing a central point of access for customers, companies, and company systems.

Understanding the customer. Experts from consulting agencies reported that many chatbot projects 
fail due to wrong requirements. "The reason for this is that the capabilities of chatbots are not those that the customer expects." [E2, COO, Marketing/Consulting]. E2 added that requirements (i.e. the resulting capabilities and dialogue structures of the chatbot) are often defined from the company perspective. The resulting mismatch between functionality and expectation by the customer often leads to chatbots failing to handle customer requests. Instead, companies should focus on the actual customer care requests that are already being faced as a starting point for defining chatbot requirements. Experts from call centers or customer care should be strongly included in this phase.

Embracing the handoff between chatbots and employees. Continuing the notion of a "right mindset", experts indicated that companies also often have wrong perceptions of "automating customer care" with chatbots. As a central takeaway, chatbots should not be seen as ways to replace human employees. Rather, chatbots should be seen as systems that can support and disburden human resources. For example, chatbots can pre-assess and prioritize customer requests, and also assist human employees through the automation of some sub-tasks. Companies should therefore not fear the hand-off from chatbot to human employees as a "lost automation", but rather embrace the opportunities of this hand-off. By means of predictive/prescriptive analysis, chatbot systems can use their knowledge and assessment of the case to guide employees and help to improve the efficiency and effectiveness in customer care.

Offer a seamless user experience. Using chatbots offers the advantage of offering customer care on multiple channels. Yet, experts strongly emphasized the need to implement a seamless user experience. With the rise of customer requests via different channels, especially social media, companies are facing vast amounts of distributed and heterogeneous information. Yet, the customer expects a seamless user experience. For example, customers want coherent handling of their request, regardless of the device used. Companies need to focus on integrating and synchronizing data from different channels (e.g., company websites, social media) and platforms (e.g., requests from computers and smartphones), in order to create a unified customer experience. Ensuring a seamless user experience is also especially relevant during the hand-off between chatbot and employee. From the perspective of the customer, the conversation should move forward, regardless of who the conversational partner is. If implemented correctly, the seamless user experience combined with fast response rates offered by chatbots is expected to promote customer satisfaction and customer delight by our experts.

\section{Conclusion}

Much attention has recently revolved around chatbots in customer care. While early research seems promising, e.g., regarding benefits such as faster response rates or customer delight, the question of how to implement and adopt chatbots in customer care is still not well understood. Research is needed to understand how to evolve new forms of intelligent chatbots and to help companies in adapting to this new form of customer care. To this aim, this work presents a research agenda based on interviews with experts in the field in order to guide future work from an industrial viewpoint, as current agenda-setting contributions fail to include this perspective. Also, we derived guidelines based on expert interviews to provide insights for companies seeking to implement chatbots in customer care.

Intuitively, this report is limited by the expert panel composition. First, the experts were all European, which could influence external validity. However, the majority of the experts from marketing/consulting agencies work with international clients; thus their experience also includes non-European cases and projects. Also, two of the experts from company clients $(\mathrm{E} 4, \mathrm{E} 9)$ are part of large, international companies, and could thus also contribute an international perspective. Furthermore, the results of this report are based on the personal experiences of the 17 interviewed experts, which could also affect external validity. Yet, the expert panel represents a multitude of different industries, e.g., energy industry, e-commerce or insurance industry, and brings together diverse views of experts committed to excellence in customer care. Here, the panel size also satisfies the required minimum of twelve experts needed for sufficient data saturation [29, 30].

To conclude, our expert panel and literature review showed us that there is a broad consensus that intelligent chatbots bare great potential for creating value for companies in customer care. While we see the existing research as highly important, our literature review also indicates that the industrial perspective should be more strongly included in identifying needed research. In this way, practice-based requirements can provide insights that may not have been captured in scientific literature, and thus advance the IS research discipline. This could also help to advocate such practice-based research as key information sources for experts.

\section{Acknowledgements}

We thank Sascha Böhr and Thomas Berg (representing 247GRAD Gmbh), and all industrial partners for insights and participation in this research. 


\section{References}

[1] S. Cook, Measuring customer service effectiveness. Routledge, 2017.

[2] R. Zemke and D. Schaaf, The service edge: 101 companies that profit from customer care. Plume Books, 1990.

[3] T. Hu, A. Xu, Z. Liu, Q. You, Y. Guo, V. Sinha, J. Luo, and R. Akkiraju, "Touch your heart: A tone-aware chatbot for customer care on social media," in Proceedings of the 2018 CHI Conference on Human Factors in Computing Systems, p. 415, ACM, 2018.

[4] F. Imgrund, M. Fischer, C. Janiesch, and A. Winkelmann, "Approaching digitalization with business process management," Proceedings of the MKWI, pp. 1725-1736, 2018.

[5] J. Baer, Hug Your Haters: How to Embrace Complaints and Keep Your Customers. Penguin, 2016.

[6] A. Følstad and P. B. Brandtzæg, "Chatbots and the new world of hci.," interactions, vol. 24, no. 4, pp. 38-42, 2017

[7] B. Zarouali, E. Van den Broeck, M. Walrave, and K. Poels, "Predicting consumer responses to a chatbot on facebook," Cyberpsychology, Behavior, and Social Networking, vol. 21, no. 8, pp. 491-497, 2018.

[8] P. B. Brandtzaeg and A. Følstad, "Why people use chatbots," in International Conference on Internet Science, pp. 377-392, Springer, 2017.

[9] S. Diederich, A. B. Brendel, and L. M. Kolbe, "On conversational agents in information systems research: Analyzing the past to guide future work,"

[10] A. Rahman, A. Al Mamun, and A. Islam, "Programming challenges of chatbot: Current and future prospective," in 2017 IEEE Region 10 Humanitarian Technology Conference (R10-HTC), pp. 75-78, IEEE, 2017.

[11] H. Van der Aa, J. Carmona Vargas, H. Leopold, J. Mendling, and L. Padró, "Challenges and opportunities of applying natural language processing in business process management," in COLING 2018: The 27th International Conference on Computational Linguistics. Proceedings of the Conference: August 20-26, 2018 Santa Fe, New Mexico, USA, pp. 2791-2801, Association for Computational Linguistics, 2018.

[12] Y. Levy and T. J. Ellis, "A systems approach to conduct an effective literature review in support of information systems research.," Informing Science, vol. 9, 2006.

[13] J. Vom Brocke, A. Simons, B. Niehaves, K. Riemer, R. Plattfaut, A. Cleven, et al., "Reconstructing the giant: On the importance of rigour in documenting the literature search process.," in European Conference on Information Systems, 2009

[14] J. vom Brocke, A. Simons, K. Riemer, B. Niehaves, R. Plattfaut, and A. Cleven, "Standing on the shoulders of giants: Challenges and recommendations of literature search in information systems research.," Communications of the Association for Information Systems, vol. 37, p. 9, 2015.

[15] G. Riccardi, "Towards healthcare personal agents," in Proceedings of the 2014 Workshop on Roadmapping the Future of Multimodal Interaction Research including Business Opportunities and Challenges, pp. 53-56, ACM, 2014
[16] H. Io and C. Lee, "Chatbots and conversational agents: A bibliometric analysis," in 2017 IEEE International Conference on Industrial Engineering and Engineering Management (IEEM), pp. 215-219, IEEE, 2017.

[17] R. N. Bolton, J. R. McColl-Kennedy, L. Cheung, A. Gallan, C. Orsingher, L. Witell, and M. Zaki, "Customer experience challenges: bringing together digital, physical and social realms," Journal of Service Management, vol. 29, no. 5, pp. 776-808, 2018.

[18] A. Følstad, M. Skjuve, and P. B. Brandtzaeg, "Different chatbots for different purposes: Towards a typology of chatbots to understand interaction design," in International Conference on Internet Science, pp. 145-156, Springer, 2018.

[19] C. Rzepka and B. Berger, "User interaction with ai-enabled systems: A systematic review of IS research," in Proceedings of the International Conference on Information Systems - Bridging the Internet of People, Data, and Things, ICIS 2018, San Francisco, CA, USA, December 13-16, 2018, 2018.

[20] M. Siddike, A. Kalam, and Y. Kohda, "Towards a framework of trust determinants in people and cognitive assistants interactions," in Proceedings of the 51st Hawaii International Conference on System Sciences, 2018.

[21] N. Tavanapour and E. A. C. Bittner, "Automated facilitation for idea platforms: Design and evaluation of a chatbot prototype," in Proceedings of the International Conference on Information Systems - Bridging the Internet of People, Data, and Things, ICIS 2018, San Francisco, CA, USA, December 13-16, 2018, 2018.

[22] J. Wirtz, P. G. Patterson, W. H. Kunz, T. Gruber, V. N. Lu, S. Paluch, and A. Martins, "Brave new world: service robots in the frontline," Journal of Service Management, vol. 29, no. 5, pp. 907-931, 2018.

[23] R. Lechler, E. Stoeckli, R. Rietsche, and F. Uebernickel, "Looking beneath the tip of the iceberg: the two-sided nature of chatbots and their roles for digital feedback exchange," in 27th European Conference on Information Systems - Information Systems for a Sharing Society, ECIS 2019, Stockholm and Uppsala, Sweden, June 8-14, 2019, 2019.

[24] L. Piccolo, M. Mensio, and H. Alani, "Chasing the chatbots: Directions for interaction and design research," 2019.

[25] C. Boyce and P. Neale, "Conducting in-depth interviews: A guide for designing and conducting in-depth interviews for evaluation input. pathfinder international," Monitoring and Evaluation, vol. 2, 2006.

[26] B. DiCicco-Bloom and B. F. Crabtree, "The qualitative research interview," Medical education, vol. 40, no. 4 , pp. 314-321, 2006.

[27] V. A. Thurmond, "The point of triangulation," Journal of nursing scholarship, vol. 33, no. 3, pp. 253-258, 2001.

[28] J. Saldaña, "Goodalls verbal exchange coding: An overview and example," Qualitative Inquiry, vol. 22, no. 1, pp. 36-39, 2016.

[29] G. Guest, A. Bunce, and L. Johnson, "How many interviews are enough? an experiment with data saturation and variability," Field methods, vol. 18, no. 1, pp. 59-82, 2006.

[30] R. Galvin, "How many interviews are enough? do qualitative interviews in building energy consumption research produce reliable knowledge?, Journal of Building Engineering, vol. 1, pp. 2-12, 2015. 